\title{
Where do AMS-02 anti-helium events come from?
}

\author{
Vivian Poulin ${ }^{* a \dagger}$, Pierre Salati ${ }^{b}$, llias Cholis ${ }^{c}$, Marc Kamionkowski $^{d}$, Joseph Silk ${ }^{d, e, f}$ \\ ${ }^{a}$ Laboratoire Univers \& Particules de Montpellier (LUPM), CNRS \& Université de Montpellier \\ (UMR-5299), Place Eugène Bataillon, F-34095 Montpellier Cedex 05, France \\ ${ }^{b}$ LAPTh, Université Savoie Mont Blanc \& CNRS, 74941 Annecy Cedex, France \\ ${ }^{c}$ Department of Physics, Oakland University, Rochester, MI 48309, USA \\ ${ }^{d}$ Department of Physics and Astronomy, Johns Hopkins University, Baltimore, MD 21218, USA \\ ${ }^{e}$ Sorbonne Universités, UPMC Univ. Paris 6 et CNRS, UMR 7095, Institut dâǍ́Astrophysique \\ de Paris, 98 bis bd Arago, 75014 Paris, France \\ ${ }^{f}$ Beecroft Institute of Particle Astrophysics and Cosmology, Department of Physics, University of \\ Oxford, Denys Wilkinson Building, 1 Keble Road, Oxford OX1 3RH, UK \\ E-mail: vivian.poulineumontpellier.fr
}

We discuss the origin of the anti-helium-3 and -4 events possibly detected by AMS-02. Using upto-date semi-analytical tools, we show that spallation from primary hydrogen and helium nuclei onto the ISM predicts a $\overline{{ }^{3} \mathrm{He}}$ flux typically one to two orders of magnitude below the sensitivity of AMS-02 after 5 years, and a $\overline{{ }^{4} \mathrm{He}}$ flux roughly 5 orders of magnitude below the AMS-02 sensitivity. We argue that dark matter annihilations face similar difficulties in explaining this event. We then entertain the possibility that these events originate from anti-matter-dominated regions in the form of anti-clouds or anti-stars. In the case of anti-clouds, we show how the isotopic ratio of anti-helium nuclei might suggest that BBN has happened in an inhomogeneous manner, resulting in anti-regions with a anti-baryon-to-photon ratio $\bar{\eta} \simeq 10^{-3} \eta$. We discuss properties of these regions, as well as relevant constraints on the presence of anti-clouds in our Galaxy. We present constraints from the survival of anti-clouds in the Milky-Way and in the early Universe, as well as from $\mathrm{CMB}$, gamma-ray and cosmic-ray observations. In particular, these require the anticlouds to be almost free of normal matter. We also discuss an alternative where anti-domains are dominated by surviving anti-stars. We suggest that part of the unindentified sources in the 3FGL catalog can originate from anti-clouds or anti-stars. AMS-02 and GAPS data could further probe this scenario.

European Physical Society Conference on High Energy Physics - EPS-HEP2019 -

10-17 July, 2019

Ghent, Belgium

\footnotetext{
* Speaker.

${ }^{\dagger}$ I want to thank Pierre Salati warmly for his help in preparing this talk. I also thank all co-authors of Ref. [1] for their participation in this study.
} 


\section{Introduction}

The origin of cosmic ray (CR) anti-matter is one of the many conundrums that AMS-02 is trying to solve thanks to precise measurements of CR fluxes at the Earth. Strikingly, AMS-02 has recently reported the possible discovery of eight anti-helium events in the mass region from 0 to $10 \mathrm{GeV} / \mathrm{c}^{2}$ with $Z=2$ and rigidity $<50 \mathrm{GV}$ [2]. Six of the events are compatible with being anti-helium-3 and two events with anti-helium-4. The total event rate is roughly one anti-helium in a hundred million heliums. This preliminary sample includes one event with a momentum of $32.6 \pm 2.5 \mathrm{GeV} / \mathrm{c}$ and a mass of $3.81 \pm 0.29 \mathrm{GeV} / \mathrm{c}^{2}$ compatible with that of anti-helium-4. Earlier already, another event with a momentum of $40.3 \pm 2.9 \mathrm{GeV}$ and a mass compatible with antihelium-3 had been reported [3]. In this proceeding, we discuss various possibilities for the origin of AMS-02 anti-helium events. Should these events be confirmed, their detection would be a breakthrough discovery, with immediate and considerable implications onto our current understanding of cosmology. The discovery of a single anti-helium-4 nucleus is challenging to explain in terms of known physics. The paper is structured as follows. Section 2 is devoted to a thorough re-evaluation of the secondary astrophysical component from spallation within the coalescence scheme. In particular, we provide the first estimate of the $\overline{{ }^{4} \mathrm{He}}$ flux at the Earth coming from the spallation of primary CR onto the ISM. A discussion on the possible limitations of our estimates and on the DM scenario is also provided. We then discuss the implications of the anti-helium observation. We essentially suggest that the putative detection of $\overline{{ }^{3} \mathrm{He}}$ and ${ }^{\overline{4} \mathrm{He}}$ by AMS-02 indicates the existence of an anti-world, i.e., a world made of anti-matter, in the form of anti-stars or anti-clouds. In section 3, properties and constraints on anti-clouds and anti-stars are presented. Finally, we draw our conclusions in sec. 4 .

\section{Updated calculation of $\bar{d}, \overline{{ }^{3} \mathrm{He}}$ and $\overline{{ }^{4} \mathrm{He}}$ from spallation onto the ISM}

As for any secondaries, the prediction of the $\overline{{ }^{3} \mathrm{He}}$ flux at Earth is the result of two main processes affected by potentially large uncertainties: i) the production due to spallation of primary CR onto the ISM and ii) the propagation of cosmic rays in the magnetic field of our Galaxy, eventually modulated by the impact of the Sun. To deal with propagation, we adapt the code developed in Refs. $[4,5]$. We model the Galaxy as a thin disk embedded in a 2D cylindrical (turbulent) magnetic halo and solve semi-analytically the full transport equation for a charged particle. We include all relevant effects, namely diffusion, diffusive reacceleration, convection, energy losses, $\overline{\mathrm{A}}$ annihilation and tertiary production. All details on our treatment of propagation can be found in Ref. [1]. Here we review the computation of the source term for anti-nuclei in the coalescence scenario.

The spallation production cross-section of an anti-nucleus A from the collision of a primary $\mathrm{CR}$ species $i$ onto an ISM species $j$ can be computed within the coalescence scenario as follows:

$$
\frac{E_{A}}{\sigma_{i j}} \frac{d^{3} \sigma_{A}^{i j}}{d^{3} k_{A}}=B_{A} \cdot\left(\frac{E_{p}}{\sigma_{i j}} \frac{d^{3} \sigma_{p}^{i j}}{d^{3} k_{p}}\right)^{Z} \cdot\left(\frac{E_{n}}{\sigma_{i j}} \frac{d^{3} \sigma_{n}^{i j}}{d^{3} k_{n}}\right)^{A-Z},
$$

where $\sigma^{i j}$ is the total inelastic cross section for the $i j$ collision, and the constituent momenta are taken at $k_{p}=k_{n}=k_{A} / A$. $B_{A}$ is the coalescence factor, whose role is to capture the probability for 
A anti-nucleons produced in a collision to merge into a composite anti-nucleus. It is often written as

$$
B_{A}=\left(\frac{4 \pi}{3} \frac{p_{\mathrm{coal}}^{3}}{8}\right)^{A-1} \frac{m_{A}}{m_{p}^{Z} m_{n}^{A-Z}},
$$

where $p_{\text {coal }}$ is the diameter of a sphere in phase-space within which anti-nucleons have to lie in order to form an anti-nucleus. The coalescence factor $B_{A}$ is a key quantity which can be estimated from $p p$-collision data, as has been done recently by the ALICE collaboration [6] for anti-deuteron and anti-helium. We use the values measured at low transverse momentum as these are adequate for CR spallation, namely $B_{2} \simeq(15 \pm 5) \times 10^{-2} \mathrm{GeV}^{2}$ and $B_{3} \simeq(2 \pm 1) \times 10^{-4} \mathrm{GeV}^{4}$. We extrapolate these values to $p A$ and $A A$ collisions. There is no measurement of $B_{4}$ yet available. Hence, we make use of eq. 2.2 in order to extract the coalescence momentum (common to each species in the coalescence model) from the $B_{3}$ measurement. This gives a coalesence momentum that varies between $0.218 \mathrm{GeV}$ and $0.262 \mathrm{GeV}$. Using the measurement of $B_{2}$, the coalescence momentum varies between $0.208 \mathrm{GeV}$ and $0.262 \mathrm{GeV}$, which is in excellent agreement with the value extracted from $B_{3}$. We stress that the fact that the coalescence momenta extracted from both coalescence factors agree is far from trival. It indicates that the coalescence scenario is much more predictive and accurate than one might have naively expected from its apparent simplicity. To phrase this otherwise: from the $B_{2}$ measurement of ALICE, one can predict how many anti-helium-3 ALICE should measure; this turns out to be in very good agreement with the actual measurement, which is quite remarkable. The final step is thus to apply eq. 2.2 to the case of anti-helium-4. We find that $B_{4}$ varies between $7.7 \times 10^{-7} \mathrm{GeV}^{6}$ and $3.9 \times 10^{-6} \mathrm{GeV}^{6}$. To impose energy conservation, we evaluate cross-sections decreasing the available center of mass energy $\sqrt{s}$ by $2 E_{p}^{*}$ for each subsequent produced anti-nucleon where $E_{p}^{*}$ denotes the anti-nucleon energy in the center of mass frame of the collision [7]. We assume that anti-neutron and anti-proton production cross-sections are equals (i.e. isospin symmetry holds).

The secondary source term can then be readily computed as:

$$
Q_{\mathrm{sec}}^{i j}\left(E_{A}\right)=4 \pi n_{j} \int_{E_{\mathrm{th}}}^{\infty} d E_{i} \phi_{i}\left(E_{i}\right) \frac{d \sigma_{A}^{i j}}{d E_{A}}\left(E_{i}, E_{A}\right) \text {, with } \frac{d \sigma_{A}^{i j}}{d E_{A}}=2 \pi k_{A} \int_{-1}^{1}\left\{E_{A} \frac{d^{3} \sigma_{A}^{i j}}{d^{3} k_{A}}\right\} d(-\cos \theta) .
$$

We assume the density of target hydrogen and helium in the ISM $n_{j}$ to be $0.9 \mathrm{~g} / \mathrm{cm}^{3}$ and 0.1 $\mathrm{g} / \mathrm{cm}^{3}$ respectively and make use of the demodulated flux of hydrogen and helium from AMS-02 with Fisk potential $730 \mathrm{MV}$. To calculate the contribution of the main channel $p+p \rightarrow \bar{A}+X$, we make use of the recent $p+p \rightarrow \bar{p}+X$ cross-section parameterization from ref. [10]. In order to incorporate other production channels (i.e. from spallation of and onto ${ }^{4} \mathrm{He}$ ), we make use of scaling relations derived in ref. [11] and multiply the $p+p \rightarrow \bar{p}+X$ cross section by $\left(A_{T} A_{P}\right)^{2.2 / 3}$ where $A_{P}$ and $A_{T}$ are the nucleon numbers of the projectile and target nuclei. The result of our computation is plotted in Fig. 1, left panel. A nice feature of the coalescence scenario is that it naturally predicts, for simple kinematic reasons, a hierarchical relation between the flux of $\bar{p}, \bar{d}$, $\overline{{ }^{3} \mathrm{He}}$ and $\overline{4} \mathrm{He}$, where each subsequent nucleus gets suppressed by a factor $10^{-4}-10^{-3}$. In Fig. 1 , right panel, we show the secondary prediction on anti-helium-3 and -4 compared to the advocated sensitivity of AMS-02 after 5 years [9]. In principle, we should compare our prediction to the measured flux, but this one is not available. Still, we can deduce from the claimed ratio of $\overline{\mathrm{He}} / \mathrm{He} \sim$ 

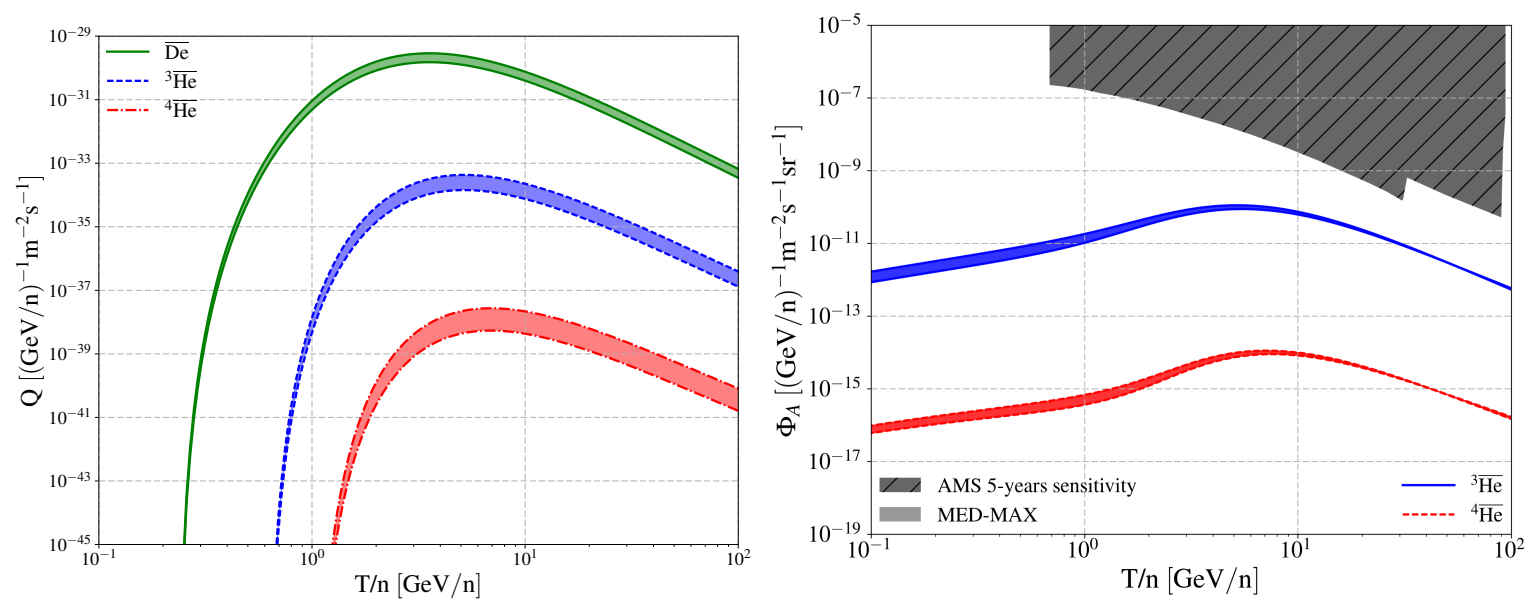

Figure 1: Left panel: Local source term for the secondary production of $\bar{d}, \overline{{ }^{3} \mathrm{He}}$ and $\overline{{ }^{4} \mathrm{He}}$. The width of the prediction represents the uncertainty on the coalescence parameter $B_{A}$. Right panel: Predicted secondary flux of ${ }^{3} \overline{\mathrm{He}}$ and ${ }^{4} \overline{\mathrm{He}}$ using the upper limit on the coalescence momentum deduced from the ALICE experiment and showing the uncertainty associated to the MED to MAX propagation model from Ref. [8]. We also show the expected sensitivity from AMS-02 [9].

$10^{-8}$ that this flux is larger by a factor of $\sim 10$ than the advocated sensitivity of AMS-02 after 5 years around $10 \mathrm{GeV}$. Hence, we confirm that it is very challenging to explain the potential AMSO2 anti-He signal as a pure secondary component. The ${ }^{\overline{3} \mathrm{He}}$ is typically one to two orders of magnitude below the sensitivity of $A M S$ - 02 after 5 years, and the $\overline{{ }^{4} \mathrm{He}}$ is roughly 5 orders of magnitude below AMS-02 sensitivity. Our results are in very good agreement with Ref. [12] and Ref. [13] when considering similar values of $B_{A}$. Note that DM face similar difficulties to explain the events (see Refs. [14, 12] for a recent reanalysis). Indeed, similarly to secondary production, one expects a hierarchical relation between the fluxes of $\bar{p}, \bar{d}, \overline{{ }^{3} \mathrm{He}}$ and ${ }^{\overline{4} \mathrm{He}}$. According to Refs. [14, 12], if DM is responsible for AMS-02 events, it seems unlikely to observe $\overline{{ }^{3} \mathrm{He}}$ without seeing a single $\bar{d}$ or overshooting $\bar{p}$ data.

\section{3. $\overline{{ }^{3} \mathrm{He}}$ and $\overline{{ }^{4} \mathrm{He}}$ as an indication for an anti-world}

Motivated by the $\overline{{ }^{3} \mathrm{He}}$ and $\overline{{ }^{4} \mathrm{He}}$, in this section we discuss the possibility that extended regions made of anti-matter have survived in our Galactic environment. The two possible cases that regions of anti-matter are present in our Galactic environment, are i) as ambient anti-matter mixed with regular matter in the ISM or in the form of anti-clouds; ii) in the form of anti-stars.

Properties of hypothetical anti-clouds: The isotopic ratio of anti-helium can potentially carry information about the physical conditions (in particular anti-matter and matter densities) within these regions. Standard big-bang nucleosynthesis (BBN) predicts the presence of many more ${ }^{4} \mathrm{He}$ events, compared to ${ }^{3} \mathrm{He}$. For normal matter, the isotopic ratio of ${ }^{4} \mathrm{He}:{ }^{3} \mathrm{He}$ is roughly $10^{4}: 1$. Within CRs, the isotopic ratio is higher since ${ }^{3} \mathrm{He}$ can be produced through spallation of ${ }^{4} \mathrm{He}$ and, according to PAMELA [15], it reaches $\sim 5: 1$ at a few $\mathrm{GeV} / \mathrm{n}$. Still, this is much lower than the possible 
measurement from AMS-02. As argued previously, increasing spallation by an order of magnitude is not realistic as it would affect all secondary species equally and lead to an over-prediction of $\bar{p}$ s. Hence, inverting the isotopic ratio requires the presence of anisotropic $\mathrm{BBN}$ in regions where the (anti)-baryon-to-photon ratio strongly differs from that measured by Planck [16]. We therefore re-calculated the BBN yields for a large number of $\eta$ values using the $\mathrm{BBN}$ code AlterBBN[17] assuming CP-invariance for simplicity (Similar results are obtained with the BBN public code PRIMAT[18]). We show in fig. 2 the number density of $\overline{\mathrm{H}}, \overline{\mathrm{D}}$ and $\overline{{ }^{4} \mathrm{He}}$ normalized to $\overline{{ }^{3} \mathrm{He}}$ as a function of the (anti)baryon-to-photon ratio $\bar{\eta}$. The width of the band features the nuclear rate uncertainties. It is possible then to obtain the right isotopic ratio (i.e. roughly $\overline{{ }^{4} \mathrm{He}}: \overline{{ }^{3} \mathrm{He}}$ of $1: 3$ ) for $\bar{\eta} \simeq$ $1.3-6 \times 10^{-13}$.

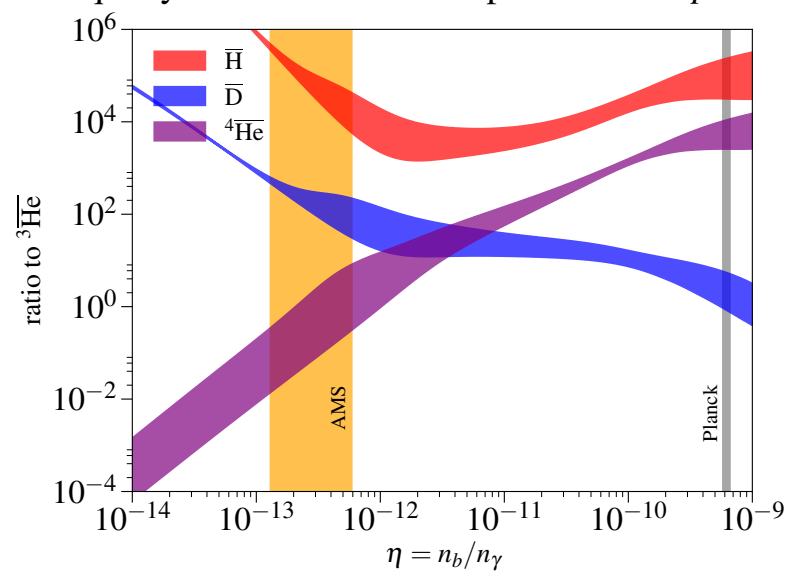

Figure 2: Abundance of $\overline{\mathrm{H}}, \overline{\mathrm{D}}$ and $\overline{{ }^{4} \mathrm{He}}$ with respect to that of $\overline{3} \mathrm{He}$ as a function of the (anti-)baryon-to-photon ratio $\bar{\eta}$. The Planck value is represented by the grey band. The value required by the $A M S-02$ experiment is shown by the orange band.

We also point out that while $\eta$ from Planck refers to an average over the whole observable Universe, $\bar{\eta}$ is based on the isotopic ratio of $\overline{{ }^{4} \mathrm{He}}: \overline{{ }^{3} \mathrm{He}}$ and is therefore a local quantity. Depending on the object from which anti-helium events are originating, it is conceivable that this number varies from place to place even within our Galaxy such that on average the isotopic ratio of $\overline{{ }^{4} \mathrm{He}}: \overline{{ }^{3} \mathrm{He}}$ is as measured.

Number and density of anti-clouds: One can get information about the anti-cloud regions from the ratio $\phi_{\overline{\mathrm{He}}} / \phi_{\mathrm{He}}$ (integrated over all energies), assuming that it reflects the ratio of the abundance of $\overline{\mathrm{He}}$ to $\mathrm{He}$ in the ISM (i.e. acceleration and propagation of CR are identical for matter and antimatter). In Ref. [1], we show that if one assumes that anti-matter forms spherical a number $N_{\bar{c}}$ of anti-clouds of radius $r_{\overline{\mathrm{c}}}$, one can estimate the number density of anti-baryons in our galaxy

$$
n_{\bar{b}} \simeq 10^{5}-10^{6.5} N_{\overline{\mathrm{c}}}^{-1}\left(\frac{n_{b}}{1 \mathrm{~cm}^{-3}}\right)\left(\frac{r_{\bar{c}}}{1 \mathrm{pc}}\right)^{-3} \mathrm{~cm}^{-3} .
$$

This key relation mostly relies on $A M S-02$ data and knowledge about Galactic properties. The only theoretical assumption is that the isotopic ratio of anti-helium is derived from BBN. From the exotic BBN we have additionally derived that at the time of BBN, $\bar{\eta} / \eta=n_{\bar{b}} / n_{b} \sim 10^{-3.5}-$ $10^{-3}$. If this ratio still holds today, it would imply that there are $N_{\bar{c}} \sim 10^{8}-10^{10}\left(r_{\bar{c}} / 1 \mathrm{pc}\right)^{-3}$ anticlouds in our Galaxy. The higher end of $N_{\overline{\mathrm{c}}}$ is close to the situation where the anti-clouds are connected in the ISM. However, this probably strongly overestimates the number of such objects, as cosmological evolution can affect these regions (and in particular the ratio $n_{\bar{b}} / n_{b}$ ) compared to primordial conditions. More realistically, AMS measured events would originate from a few highly-dense clouds.

Survival time of anti-clouds: We can gain information about the properties of the anti-matter regions and in particular constrain the amount of normal matter within them by estimating the typical lifetime of anti-matter in our Galaxy. The lifetime depends on the relative velocity between 
matter and anti-matter particles, as the annihilation cross-section can be strongly enhanced at lowvelocity. For our estimates, we will follow the parameterization suggested in Ref. [19]. The survival rate depends on whether anti-matter is in the form of cold clouds, where $T \sim \mathscr{O}(30) \mathrm{K}$, or in hot ionized clouds, where $T \sim \mathscr{O}\left(10^{6}\right) \mathrm{K}$. By requiring $\tau_{\mathrm{ann}}>t_{\mathrm{gal}} \simeq 2.8 \times 10^{17} \mathrm{~s}$, one gets

$$
n_{p}^{\text {cold }}<3.5 \times 10^{-8} \mathrm{~cm}^{-3}, n_{p}^{\text {hot }}<6.1 \times 10^{-5} \mathrm{~cm}^{-3} .
$$

for such anti-clouds to survive in our Galaxy. Note that these numbers are independent of the size and density of anti-matter regions and agrees well with Refs. $[19,20]$. We conclude from this short analysis that anti-matter would survive in our Galaxy only if there is some separation between the species, in which case it could be a viable candidate to explain the anti-helium events. However, diffuse anti-matter occupying all the volume of our Galaxy would not survive over the life span of our Galaxy. Additionally, we can perform the same calculation in the early Universe, splitting between three periods depending on the annihilation regime. We find

$\frac{n_{p}^{\text {local }}}{n_{p}^{\text {cosmo }}}\left(z>z_{\mathrm{BBN}}\right)<\left(\frac{67}{1+z}\right), \frac{n_{p}^{\text {local }}}{n_{p}^{\text {cosmo }}}\left(z_{\mathrm{eq}}<z<z_{\mathrm{BBN}}\right)<\left(\frac{1.7 \times 10^{-3}}{\sqrt{1+z}}\right), \frac{n_{p}^{\text {local }}}{n_{p}^{\text {cosmo }}}\left(z<z_{\mathrm{eq}}\right)<\frac{6.3 \times 10^{-2}}{(1+z)^{3 / 2}}$.

This confirms that anti-matter must have formed in regions where the density of protons was much lower than the cosmological average (at least $\mathscr{O}\left(10^{-8}\right)$ if these regions form just at the start $\mathrm{BBN}$ ), such that annihilations only occur at the border of the anti-matter dominated domains. However, we expect galaxy formation to have mixed up partially the species. Hence, it makes the existence of such anti-clouds rather improbable. It is possible to get even stronger constrains by using the (non-)observations of gamma-ray from $p \bar{p}$-annihilation (see Ref. [1]).

Properties of anti-stars from AMS-02 measurement: An alternative possibility is that the antimatter is in the form of stars. This is likely more realistic, since anti-stars would naturally be free of matter at their heart, and annihilation are limited to their surface. The typical scenario, already suggested in Ref. [21], and more recently in Ref. [22] is that the anti-star are formed from a very dense clump within an anti-matter domain, which could have survived since the early Universe. $\mathrm{BBN}$ in a very dense medium would result in the creation of very large amounts of $\overline{{ }^{4} \mathrm{He}}$, so that the anti-star could be largely dominated by $\overline{{ }^{4} \mathrm{He}}$. Difficulties associated to this scenario are two fold: i) a mechanism responsible for the acceleration of $\overline{{ }^{4} \mathrm{He}}$ up to $50 \mathrm{GeV}$ energy is required; ii) the isotopic ratio $\overline{{ }^{4} \mathrm{He}}: \overline{{ }^{3} \mathrm{He}}$ must be inverted during propagation close to the source.

Depending on the answer to point i), the estimate of the number of such objects can largely vary. A single close-by anti-star might be responsible for the entire anti-helium flux seen by AMS-02. To quantify, if a fraction $f_{\text {acc }}$ of a single anti-star experienced such an event, the total amount of anti-helium ejected in the Galaxy would be approximately

$\Phi_{\overline{\mathrm{He}}}=\left(\frac{c}{V_{\mathrm{gal}}}\right)\left(\frac{f_{\overline{\mathrm{He}}} M_{\bar{*}}}{m_{\overline{\mathrm{He}}}}\right) f_{\mathrm{acc}} \simeq 10^{-9}\left(\frac{(4 \pi / 3)(10 \mathrm{kpc})^{3}}{V_{\mathrm{gal}}}\right)\left(\frac{M_{\bar{*}}}{M_{\odot}}\right)\left(\frac{f_{\mathrm{acc}}}{10^{-8}}\right)\left(\frac{f_{\overline{\mathrm{He}}}}{1}\right) \# \overline{\mathrm{He}} \mathrm{cm}^{-2} \mathrm{~s}^{-1}$,

where $f_{\overline{\mathrm{He}}}$ represents the fraction of anti-helium- 4 within the anti-star. Interestingly, for $f_{\overline{\mathrm{He}}}=1$ and $f_{\text {acc }}=10^{-8}$, this is in good agreement with the measured AMS-02 flux in the GeV range. However, given that $\mathrm{CR}$ nuclei stay confined within the magnetic halo over a timescale ranging from $\sim 10^{7}$ 
to $3 \times 10^{8} \mathrm{yr}$, which is short compared to the $\sim 10^{10} \mathrm{yr}$ of existence of our Galaxy, the probability that such an event occured nowadays is smaller than $3 \%$, and it is therefore more likely that there exists a population of such stars. If anti-stars are formed in star clusters, conventional acceleration mechanisms (e.g. SN shock-waves, jets, outflows) can also be responsible for CRs anti-helium at such energies. We note that massive stars leading to SN explosions are short-lived, and therefore primordial anti-stars would most likely not survive over the course of the Universe. On the other hand, a binary of anti-matter white dwarfs would merge giving an anti-matter type Ia supernova. Regular white-dwarf mergers occur at a rate per unit stellar mass of $1.4 \times 10^{-13} \mathrm{yr}^{-1} \mathrm{M}_{\odot}^{-1}$ [23]. Requiring that at least one binary of anti-matter white dwarfs merges over a typical CR diffusion timescale translates into a minimal stellar population of anti-stars of $\sim 2.4 \times 10^{4}$ to $7 \times 10^{5} \mathrm{M}_{\odot}$ within $10 \mathrm{kpc}$ from the Earth. This is very small compared to the Galactic stellar population which amounts to $\sim 6 \times 10^{10} \mathrm{M}_{\odot}$. In order to achieve point ii), spallation around the source needs to be efficient enough such as to convert a large amount of $\overline{{ }^{4} \mathrm{He}}$ into $\overline{{ }^{3} \mathrm{He}}$. Given the total cross-section for $\bar{p}^{4} \mathrm{He}$ interactions as well as the fraction of events going into ${ }^{3} \mathrm{He}+X$ measured by the Lear collaboration [24], we estimate that a grammage of order $20 \mathrm{~g} / \mathrm{cm}^{2}$ would be enough to generate an isotopic ratio ${ }^{3 \mathrm{He}}: \overline{{ }^{4} \mathrm{He}}$ of roughly $3: 1$. If true, the origin of this grammage woud most likely be related to the origin of the anti-star itself. Indeed, we expect anti-stars to be surrounded by much denser material than that around normal stars, as the former are born from large over-densities at a much earlier time.

Given that a single anti-star could explain $A M S-02$ data, there is no strong constrain on the presence of such objects in our Galaxy. Indeed, even if all of the anti-helium- 4 is converted to antiprotons, it would only lead to a handful of events that can easily be hidden within the $\sim 10^{5} \bar{p}$ events observed by $A M S-02$ [25]. Assuming spherical (Bondi) accretion and making use of unidentified source in the 2FGL Fermi-LAT catalog, Ref. [26] constrained the local environment, within $150 \mathrm{pc}$ from the Sun, to have $N_{\bar{*}}<4 \times 10^{-5} N_{*}$. The brightest unassociated source from the 3FGL catalog emits $2 \times 10^{-8} \# \gamma \mathrm{cm}^{-2} \mathrm{~s}^{-1}$ above $1 \mathrm{GeV}$ [27]. From this, we can estimate the distance of the closest antistar assuming that its luminosity is sourced by annihilation at its surface. The luminosity associated to the emission is

$$
L_{\bar{*}}=8 \pi R_{\bar{*}}^{2} v n_{p} \simeq 10^{31}\left(\frac{R_{\bar{*}}}{10^{11} \mathrm{~cm}}\right)^{2}\left(\frac{v}{300 \mathrm{~km} \mathrm{~s}^{-1}}\right)\left(\frac{n_{p}}{1 \mathrm{~cm}^{-3}}\right) \# \gamma \mathrm{s}^{-1},
$$

where we assumed that the dominant channel for prompt photon emission is through $\pi_{0}$ production (whose average multiplicity is 2 per annihilation at rest [28]). The minimal distance of such an object is obtained by requiring

$$
\frac{L_{\bar{*}}}{4 \pi d_{*}^{2}} \leq 2 \times 10^{-8} \# \gamma \mathrm{cm}^{-2} \mathrm{~s}^{-1}, \Rightarrow d_{\bar{*}} \geq 6 \times 10^{18} \sqrt{\left(\frac{R_{\bar{*}}}{10^{11} \mathrm{~cm}}\right)\left(\frac{v}{300 \mathrm{~km} \mathrm{~s}^{-1}}\right)\left(\frac{n_{p}}{1 \mathrm{~cm}^{-3}}\right)} \mathrm{cm} .
$$

Hence, it is possible that an anti-star whose main source of emission is annihilation at its surface lies in a close-by environment $\sim \mathscr{O}(1 \mathrm{pc})$ away from the Sun.

\section{Discussion and conclusions}

In this work, we have studied the implications of the potential discovery of anti-helium-3 
and -4 nuclei by the AMS-02 experiment. Using up-to-date semi-analytical tools, we have shown that it is impossible to explain these events as secondaries, i.e., from the spallation of CR protons and helium nuclei onto the ISM. The $\overline{{ }^{3} \mathrm{He}}$ is typically one to two orders of magnitude below the sensitivity of $A M S-02$ after 5 years, and the $\overline{{ }^{4} \mathrm{He}}$ is roughly 5 orders of magnitude below AMS-02 reach. It is conceivable that ${ }^{3} \mathrm{He}$ has been misidentified for $\overline{{ }^{4} \mathrm{He}}$. Still, we have argued that the pure secondary explanation would require a large increase of the coalescence momentum at low energies, a behavior that goes against theoretical considerations and experimental results. The DM scenario suffers the same difficulties. Hence, we have discussed how this detection, if confirmed, would indicate the existence of an anti-world, in the form of anti-stars or anti-clouds. Needless to say, the successful creation and survival of such objects within a coherent cosmological model is far from obvious. Here we just mention that there are many scenarios discussed in the literature [20, 22, 29], including the Affleck-Dine mechanism [30], which would lead to the formation of "bubbles" of matter and anti-matter with arbitrarily large values of the baryon-asymmetry locally. Depending on the relation between their mass and the corresponding Jeans mass, these bubbles can then lead to the formation of anti-star-like objects, either through specific inflation scenarios with large density contrast $[31,32]$ on scales re-entering the horizon around the QCD phase-transition, i.e., $T \sim \mathscr{O}(100 \mathrm{MeV})$, or from peculiar dynamics of the plasma within the bubble, as described for instance in Ref. [21]. Given the strong implications of the discovery of a single anti-helium4 nucleus for cosmology, important theoretical and experimental efforts must be undertaken in order to assess whether the reported events could be explained by a more mundane source, such as interactions within the detector, or another source of yet unkown systematic error. Still, this potential discovery would represent an important probe of conditions prevailing in the very early Universe and should be investigated further in future work.

\section{References}

[1] V. Poulin, P. Salati, I. Cholis, M. Kamionkowski, and J. Silk, "Where do the AMS-02 antihelium events come from?," Phys. Rev., vol. D99, no. 2, p. 023016, 2019.

[2] V. A. Choutko AMS days at la Palma, Spain, 2018.

[3] S. Ting The First Five Years of the Alpha Magnetic Spectrometer on the ISS, 2016.

[4] M. Boudaud, M. Cirelli, G. Giesen, and P. Salati, "A fussy revisitation of antiprotons as a tool for Dark Matter searches," JCAP, vol. 1505, no. 05, p. 013, 2015.

[5] G. Giesen, M. Boudaud, Y. GÃl'nolini, V. Poulin, M. Cirelli, P. Salati, and P. D. Serpico, "AMS-02 antiprotons, at last! Secondary astrophysical component and immediate implications for Dark Matter," JCAP, vol. 1509, no. 09, p. 023, 2015.

[6] S. Acharya et al., "Production of deuterons, tritons, ${ }^{3} \mathrm{He}$ nuclei and their anti-nuclei in pp collisions at $\sqrt{s}=0.9,2.76$ and $7 \mathrm{TeV}, " 2017$.

[7] P. Chardonnet, J. Orloff, and P. Salati, "The Production of antimatter in our galaxy," Phys. Lett., vol. B409, pp. 313-320, 1997.

[8] F. Donato, N. Fornengo, D. Maurin, and P. Salati, "Antiprotons in cosmic rays from neutralino annihilation," Phys. Rev., vol. D69, p. 063501, 2004.

[9] A. A. Kounine Proceedings, 32nd ICRC 2011, vol. c, p. 5, 2011. 
[10] M. di Mauro, F. Donato, A. Goudelis, and P. D. Serpico, "New evaluation of the antiproton production cross section for cosmic ray studies," Phys. Rev., vol. D90, no. 8, p. 085017, 2014.

[11] J. W. Norbury and L. W. Townsend, "Parameterized total cross-sections for pion production in nuclear collisions,” Nucl. Instrum. Meth., vol. B254, pp. 187-192, 2007.

[12] M. Korsmeier, F. Donato, and N. Fornengo, "Prospects to verify a possible dark matter hint in cosmic antiprotons with antideuterons and antihelium," 2017.

[13] K. Blum, K. C. Y. Ng, R. Sato, and M. Takimoto, "Cosmic rays, anti-helium, and an old navy spotlight," 2017.

[14] A. Coogan and S. Profumo, "Can AMS anti-Helium events come from dark matter? Maybe!," 2017.

[15] O. Adriani et al., "Measurements of Cosmic-Ray Hydrogen and Helium Isotopes with the PAMELA experiment," Astrophys. J., vol. 818, no. 1, p. 68, 2016.

[16] P. A. R. Ade et al., "Planck 2015 results. XIII. Cosmological parameters," Astron. Astrophys., vol. 594, p. A13, 2016.

[17] A. Arbey, "AlterBBN: A program for calculating the BBN abundances of the elements in alternative cosmologies," Comput. Phys. Commun., vol. 183, pp. 1822-1831, 2012.

[18] C. Pitrou, A. Coc, J.-P. Uzan, and E. Vangioni, "Precision big bang nucleosynthesis with improved Helium-4 predictions," Phys. Rept., vol. 04, p. 005, 2018.

[19] G. Steigman, “Observational tests of antimatter cosmologies," Ann. Rev. Astron. Astrophys., vol. 14, pp. 339-372, 1976.

[20] C. Bambi and A. D. Dolgov, “Antimatter in the Milky Way,” Nucl. Phys., vol. B784, pp. 132-150, 2007.

[21] A. Dolgov and J. Silk, "Baryon isocurvature fluctuations at small scales and baryonic dark matter," Phys. Rev., vol. D47, pp. 4244-4255, 1993.

[22] S. I. Blinnikov, A. D. Dolgov, and K. A. Postnov, "Antimatter and antistars in the universe and in the Galaxy,” Phys. Rev., vol. D92, no. 2, p. 023516, 2015.

[23] C. Badenes and D. Maoz, "The Merger Rate of Binary White Dwarfs in the Galactic Disk," Astrophys. J., vol. 749, p. L11, 2012.

[24] F. Balestra et al., "Inelastic Interaction of Anti-protons With ${ }^{4} \mathrm{He}$ Nuclei Between 200-MeV/c and 600-MeV/c,” Phys. Lett., vol. 165B, pp. 265-269, 1985.

[25] M. Aguilar et al., "Antiproton Flux, Antiproton-to-Proton Flux Ratio, and Properties of Elementary Particle Fluxes in Primary Cosmic Rays Measured with the Alpha Magnetic Spectrometer on the International Space Station,” Phys. Rev. Lett., vol. 117, no. 9, p. 091103, 2016.

[26] P. von Ballmoos, "Antimatter in the Universe : Constraints from Gamma-Ray Astronomy," Hyperfine Interact., vol. 228, no. 1-3, pp. 91-100, 2014.

[27] F. Acero et al., "Fermi Large Area Telescope Third Source Catalog," Astrophys. J. Suppl., vol. 218, no. 2, p. 23, 2015 .

[28] C. Amsler, "Proton - anti-proton annihilation and meson spectroscopy with the crystal barrel," Rev. Mod. Phys., vol. 70, pp. 1293-1340, 1998.

[29] M. Yu. Khlopov, R. V. Konoplich, R. Mignani, S. G. Rubin, and A. S. Sakharov, "Physical origin, evolution and observational signature of diffused antiworld," Astropart. Phys., vol. 12, pp. 367-372, 2000. 
[30] I. Affleck and M. Dine, “A New Mechanism for Baryogenesis,” Nucl. Phys., vol. B249, pp. 361-380, 1985.

[31] B. J. Carr and S. W. Hawking, "Black holes in the early Universe," Mon. Not. Roy. Astron. Soc., vol. 168 , pp. 399-415, 1974.

[32] B. J. Carr, “The Primordial black hole mass spectrum,” Astrophys. J., vol. 201, pp. 1-19, 1975. 